\title{
Assessment of variations in control of asthma over time
}

\author{
C. Combescure*, P. Chanez\#, P. Saint-Pierre*, J-P. Daurès*, H. Proudhon ", P. Godard", on behalf of \\ the Association pour la Recherche en Intelligence Artificielle ${ }^{T M}$ (ARIA) group
}

Assessment of variations in control of asthma over time. C. Combescure, P. Chanez, P. Saint-Pierre, J-P. Daurès, H. Proudhon, P. Godard, on behalf of the Association pour la Recherche en Intelligence Artificielle ${ }^{T M}$ (ARIA) group. C) ERS Journals Ltd 2003.

ABSTRACT: Control and severity of asthma are two different but complementary concepts. The severity of asthma could influence the control over time. The aim of this study was to demonstrate this relationship.

A total 365 patients with persistent asthma (severity) were enrolled and followed-up prospectively. Data were analysed using a continuous time homogeneous Markov model of the natural history of asthma. Control of asthma was defined according to three health states which were qualified: optimal, suboptimal and unacceptable control (states 1,2 and 3). Transition forces (denoted $\lambda_{i j}$ from state $i$ to state $j$ ) and transition probabilities between control states were assessed and the results stratified by asthma severity were compared.

Models were validated by comparing expected and observed numbers of patients in the different states. Transition probabilities stabilised between 100-250 days and more rapidly in patients with mild-to-moderate asthma. Patients with mild-to-moderate asthma in suboptimal or unacceptable control had a high probability of transition directly to optimal control. Patients with severe asthma had a tendency to remain in unacceptable control.

A Markov model is a useful tool to model the control of asthma over time. Severity modified clearly the health states. It could be used to compare the performance of different approaches to asthma management.

Eur Respir J 2003; 22: 298-304.
*Laboratoire de Biostatistique, Institut Universitaire de Recherche Clinique, and ${ }^{\#}$ Service de Pneumologie, CHU Hôpital Arnaud de Villeneuve, Montpellier, and "Laboratoire de Biostatistique, Hôpital La Timone, Marseille, France.

Correspondence: C. Combescure

Laboratoire de Biostatistique

Institut Universitaire de Recherche Clinique 641 Av. G. Giraud

34093 Montpellier

France

Fax: 33467542731

E-mail: combesc@iurc1.iurc.montp.inserm.fr

Keywords: Asthma

control states

Markov model

severity

Received: September 22002

Accepted after revision: February 212003

This study was supported by an unrestricted educational grant from GlaxoSmithKline, France.
Asthma is a chronic inflammatory disease of the bronchi which may resolve either spontaneously or after appropriate treatment [1]. Phenotypic characterisation of asthma is difficult. Several reports [2-4] have proposed that asthma patients be classified according to the severity of their disease. The definition of severity is based on a combination of symptoms, the degree of bronchial obstruction and the intensity of treatment. Recently, Canadian experts have recommended that the dose of inhaled corticosteroids necessary to obtain good control of asthma should be included when evaluating severity [5].

The concept of control is new [6,7] and has been the subject of several investigations [8-10]. Although asthma scores for assessing this aspect have been published in the literature [11, 12], they do not all appear to be equivalent. Such scores are based on the frequency of symptoms, their duration, the degree of bronchial obstruction and the need for rescue medication (essentially short-acting $\beta_{2}$-mimetics). Most authors agree that $8-15$ days is a sufficient period of time to evaluate asthma control, although some have expressed the results as number of days without symptoms [12]. It is obvious that severity and control of asthma are two different but complementary concepts. Their use in practice should allow optimisation of clinical management.

In 1995, the present authors' developed an "expert system" to appreciate the natural history of asthma [13]. During the validation of this system, it became apparent that it was difficult to consider the total asthmatic population as a whole.
This led to an incorporation of the concepts of severity and control, in order to better discriminate between patients. Since then, a large temporal database has accumulated prospectively with each patient being characterised according to the level of severity of asthma and the level of control at each visit.

These levels of control can be considered as health states. They can be defined into three levels, i.e. optimal, suboptimal and unacceptable. The question is to know how to predict the long-term control of asthma. For this purpose, the real-time multistate Markov model has been used, and the potential variation of control was first investigated according to the initial severity stage of the disease.

\section{Materials and methods}

\section{Patients}

Data used in this study were collected over a 3-yr period by a number of French chest physicians.

Adult asthmatic patients were enrolled prospectively. In all patients, asthma had been diagnosed for $>1$ yr and the diagnosis was confirmed using the American Thoracic Society criteria. For entry into the study, documentation of a minimum of $12 \%$ reversibility of airways obstruction during the previous year, or a history of bronchial hyperactivity for patients 
without airway obstruction, was required for all patients. Smoking was not an exclusion criterion. Asthmatic patients were treated according to international recommendations [1]. Only patients with persistent asthma and with at least two visits were included into the study.

The case-report form used to validate the expert system [13] allowed recording of data at successive follow-up visits. The data reflect the real activity of a hospital asthma visit, with patients returning at variable intervals according to their perceived needs.

The expert system has been described in detail elsewhere [13]; it consists of a case database, a knowledge base and an inferential driver. The expert system generates a variety of conclusions, including, in particular, asthma severity, which was based on symptoms and medication needs according to international and various national consensus [5]. The definition of the severity is detailed in Appendix 1.

\section{Markov model}

Figure 1 summarises the continuous homogeneous Markov model used. It allows the modelling of patient follow-up as a succession of transitions between health states over time. The states chosen were levels of control, and all transitions between the states are possible.

Control of asthma was based on the events taking place during the last month and was assigned to one of the following three levels of control: optimal control, suboptimal control and unacceptable control. The definition of the states are detailed in Appendix 2, and was adapted from [5]. It was based on the exacerbation, $\beta_{2}$-mimetic use associated with the presence of symptoms, measurement of forced expiratory volume in one second (FEV1) and dyspnoea.

The parameters $\lambda_{\mathrm{ij}}$ (fig. 1), associated with the transition from the state $\mathrm{i}$ to the state $\mathrm{j}$, which characterise the model are known as transition forces. They are interpreted as speed of transition and expressed in number of transitions per day. The models were considered homogeneous, i.e. the transition forces were independent of time. The transition probabilities from state $\mathrm{i}$ to state $\mathrm{j}$ are deduced directly from the transition forces.

The parameters were assessed by maximum likelihood [14, 15]. The parameters between mild-to-moderate and severe patients were compared by a Wald test (W). For each stratum, the parameters were compared with each other by a likelihood ratio test (LRT). The mean length of times spent in each health state before leaving are assessed from these parameters, and standard deviations are estimated using the delta method [16].

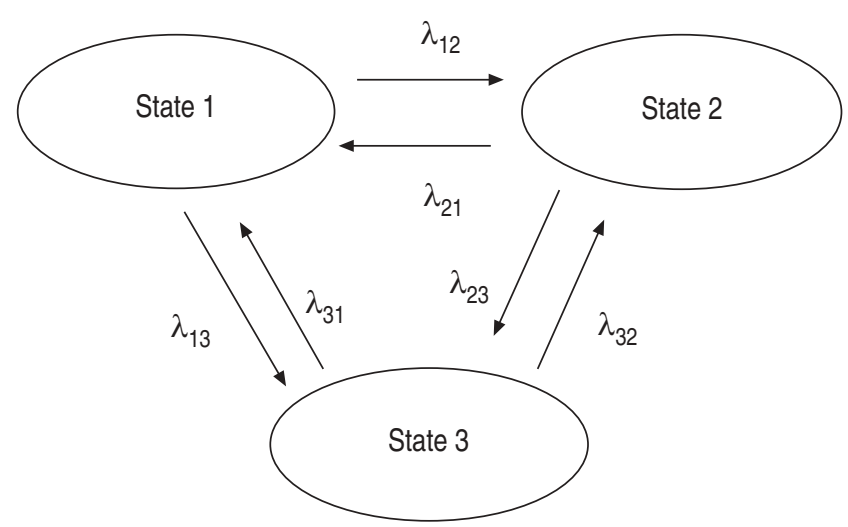

Fig. 1.-Diagram summarising the Markov model. The arrows indicate the transitions that patients could undergo. $\lambda_{\mathrm{ij}}$ are the parameters associated with transition from state "i" to state "j".
Goodness-of-fit of the homogeneous Markov models was tested with a Chi-squared test comparing the control state distribution at the last visit and the theoretical one at 100 , 200, 300, and 400 days predicted from the models.

\section{Statistical analysis}

The mean values for the sociodemographic variables according to severity score were analysed by the Kruskall Wallis (KW) or Chi-squared tests. The results are expressed as means \pm SD, with the addition of median values where they may aid interpretation.

\section{Results}

\section{Description of the patients}

A total 365 patients were included in this study between 1997-1998, and were followed up until 2001. The total number of consultations was 1,283 . The median duration of follow-up was 161 days (interquartile range: 84 406). The demographic data for these patients are summarised in table 1 .

There were 166, 116 and 83 patients in the mild, moderate or severe persistent asthma categories respectively. Several parameters were found to be correlated with asthma severity (table 1). Age differed significantly $(\mathrm{KW} ; \mathrm{p}=0.02)$ between the different states of asthma and correlated positively with severity. There were more female patients than males, but the differences in sex ratio between states were not significant. Severity increased with body mass index $(\mathrm{KW} ; \mathrm{p}=0.02)$, and also with duration of asthma $(\mathrm{KW} ; \mathrm{p}=0.03)$ and airflow impairment as represented by the FEV1 $(\mathrm{KW} ; \mathrm{p}<0.01)$.

When risk factors were examined (table 1), atopy was slightly less frequent among patients with more severe disease than in patients with mild-to-moderate asthma $(60 \%$ versus $70 \%$ ) but this difference was not statistically significant (Chisquared; $\mathrm{p}=0.20$ ). Sixty-six per cent of all patients had a history of rhinitis, but no correlation with severity was noted (Chi-squared; $\mathrm{p}=0.58$ ). The proportion of current smokers ranged from $12-15 \%$, but again there was no significant correlation with severity (Chi-squared; $\mathrm{p}=0.85$ ).

\section{Markov model in the total population}

The total number of consultations was 1,283 and could be divided into 357, 336 and 590 for control states 1, 2 and 3, respectively.

The transition forces can be defined as the speed of transition $[14,15]$ and varied between $3.4 \times 10^{-3}$ and $11.1 \times 10^{-3}$ (table 2 ). $\lambda_{12}$ and $\lambda_{21}$ were significantly larger than $\lambda_{13}$ and $\lambda_{23}$ (LRT; $\mathrm{p}=0.03$ and $\mathrm{p}<0.01$, respectively). There was no significant difference between $\lambda_{31}$ and $\lambda_{32}$ (LRT; $p=0.17$ ). Patients who changed from state 1 had a greater tendency to change to state 2 than to state 3 , and those who changed from state 2 had a greater tendency to change to state 1 than to state 3 .

The mean length of time spent in a health state before leaving it (expected from the model) was $72 \pm 7.6,66 \pm 7.1$ and $114 \pm 9.7$ days for states 1,2 and 3 , respectively.

Figure 2 demonstrates the evolution of transition probability over time. The transition probability out of states 1 or 2 became stabilised at $\sim 200$ days, whereas the transition probability out of state 3 became stabilised towards 300 days.

The number of patients in each control state was not significantly different from the theoretical distribution expected 
Table 1.-Patient characteristics at baseline examination according to asthma severity

\begin{tabular}{|c|c|c|c|c|}
\hline \multirow[t]{2}{*}{ Parameter } & \multicolumn{3}{|c|}{ Asthma severity (expert system) } & \multirow[t]{2}{*}{ p-value ${ }^{\#}$} \\
\hline & Mild & Moderate & Severe & \\
\hline Patients $\mathrm{n}$ & 166 & 116 & 83 & \\
\hline Age yrs & & & & 0.02 \\
\hline Mean \pm SD & $40 \pm 17$ & $44 \pm 15$ & $44 \pm 16$ & \\
\hline Median (range) & $39(8-79)$ & $46(17-75)$ & $45(13-77)$ & \\
\hline Sex female $\%$ & 57 & 66 & 53 & 0.12 \\
\hline BMI & & & & 0.02 \\
\hline Mean \pm SD & $23 \pm 4$ & $24 \pm 5$ & $25 \pm 5$ & \\
\hline Median (range) & $22(14-42)$ & $24(17-45)$ & $25(15-43)$ & \\
\hline Duration of asthma yrs & & & & 0.03 \\
\hline Mean \pm SD & $15 \pm 13$ & $20 \pm 15$ & $20 \pm 15$ & \\
\hline Median (range) & $13(0-64)$ & $17(0-65)$ & $16(0-63)$ & \\
\hline FEV1 \% pred & & & & $<0.01$ \\
\hline Mean \pm SD & $87 \pm 16$ & $79 \pm 18$ & $55 \pm 23$ & \\
\hline Median (range) & $87(50-130)$ & $80(45-122)$ & $48(13-128)$ & \\
\hline Atopy $\%$ & 70 & 63 & 60 & 0.20 \\
\hline Rhinitis \% & 70 & 65 & 69 & 0.58 \\
\hline Current smokers \% & 14 & 15 & 12 & 0.85 \\
\hline
\end{tabular}

\#: p-values were calculated with the Kruskal-Wallis or Chi-squared tests.

Table 2. - Estimation of transition forces in the whole population ${ }^{\#}$

\begin{tabular}{lrc}
\hline Transition forces & Value & SD \\
\hline$\lambda_{12}$ & $9.6 \times 10^{-3}$ & $1.3 \times 10^{-3}$ \\
$\lambda_{13}$ & $4.2 \times 10^{-3}$ & $0.6 \times 10^{-3}$ \\
$\lambda_{21}$ & $11.1 \times 10^{-3}$ & $1.5 \times 10^{-3}$ \\
$\lambda_{23}$ & $4.1 \times 10^{-3}$ & $0.7 \times 10^{-3}$ \\
$\lambda_{31}$ & $5.3 \times 10^{-3}$ & $0.4 \times 10^{-3}$ \\
$\lambda_{32}$ & $3.4 \times 10^{-3}$ & $0.4 \times 10^{-3}$ \\
\hline
\end{tabular}

${ }^{\#}$ : transition forces are presented as number of transitions per day.

from the model at 100, 200, 300 and 400 days (Chi-squared, $\mathrm{p}=0.41, \mathrm{p}=0.96, \mathrm{p}=0.94$ and $\mathrm{p}=0.24$ respectively).

\section{Stratification according to severity}

The study population was stratified into two subpopulations: mild-to-moderate persistent asthma and severe persistent asthma.

There were 257 patients with persistent mild-to-moderate asthma. In total these patients attended 699 consultations of whom 266, 204 and 229 fell into health states 1, 2 and 3, respectively.

The transition forces varied from $1 \times 10^{-7}-17.8 \times 10^{-3}$ (table 3 ). There was no significant difference between $\lambda_{12}$ and $\lambda_{13}$ (LRT; $\mathrm{p}=0.40) . \lambda_{21}$ was significantly larger than $\lambda_{23}(\mathrm{LRT} ; \mathrm{p}=0.01)$ and $\lambda_{31}$ was significantly larger than $\lambda_{32}$ (LRT; $\mathrm{p}<0.01$ ). Patients who changed from state 2 had a greater tendency to enter state 1 than to state 3 , and those who changed from state 3 had a greater tendency to enter state 2 than to state 1.

The mean time spent in one state before changing to

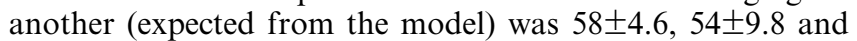
$56 \pm 2.8$ days for states 1,2 and 3 , respectively.

Figure 3 shows the evolution of transition probability over time. The probabilities of transition out of state 1 became stabilised at $\sim 100$ days, and the transition probability out of states 2 and 3 towards 200 days.

The number of patients in each control state was not significantly different from the theoretical distribution expected from the model at 200, 300 and 400 days (Chi-squared, $p=0.27$, $\mathrm{p}=0.49$ and $\mathrm{p}=0.17$ respectively). At 100 days, the difference was significant (Chi-squared, $\mathrm{p}=0.003$ ).

There were 108 patients with persistent severe asthma. These patients attended a total of 584 consultations of which 91,132 and 361 were in states 1, 2 and 3, respectively.

The transition forces varied from $3.2 \times 10^{-3}-8.3 \times 10^{-3}$ (table 3 ). There was no significant difference between $\lambda_{12}$ and $\lambda_{13}$ (LRT; $\mathrm{p}=0.91)$, between $\lambda_{21}$ and $\lambda_{23}(\mathrm{LRT} ; \mathrm{p}=0.45)$ or between $\lambda_{31}$ and $\lambda_{32}$ (LRT; $\mathrm{p}=0.67$ ).

The mean length of time spent in one health state before changing to another (expected from the model) was $63 \pm 13.5$, $84 \pm 13.1$ and $143 \pm 18.1$ days for states 1,2 and 3 , respectively.

Figure 4 shows the evolution of transition probability over time. The transition probability out of any of the three states became stable towards 300 days.

The number of patients in each control state was not significantly different from the theoretical distribution expected from the model at 100, 200, 300 and 400 days (Chi-squared; $\mathrm{p}=0.14, \mathrm{p}=0.08, \mathrm{p}=0.52$ and $\mathrm{p}=0.87$ respectively).

\section{Comparison of the two groups of patients}

The number of consultations in state 3 was significantly higher in patients with persistent severe asthma (361 consultations out of 584 versus 229 out of 699 ; Chi-squared $\mathrm{p}<0.01$ ).

The transition forces $\lambda_{12}, \lambda_{13}$ and $\lambda_{32}$ were not significantly different between the two groups of patients (table 3 ) (W; $\mathrm{p}=0.40, \mathrm{p}=0.54$ and $\mathrm{p}=0.09$, respectively). $\lambda_{21}$ and $\lambda_{31}$ were significantly higher in patients with persistent mild-to-moderate asthma ( $\mathrm{W} ; \mathrm{p}<10^{-3}$ and $\mathrm{p}<10^{-3}$, respectively). $\lambda_{23}$ was significantly smaller in patients with mild-to-moderate asthma $(\mathrm{W}$; $\left.\mathrm{p}<10^{-3}\right)$. A patient with nonsevere asthma in state 2 or 3 therefore had a tendency to change to state 1 while a patient with severe asthma in state 2 had a tendency to change to state 3. Patients with persistent severe asthma on average took more time to leave states 2 or 3 than patients with mild-tomoderate asthma (84 versus 54 days and 143 versus 56 days, respectively).

Figures 3 and 4 show that the transition probabilities became stable more quickly in patients with mild-moderate asthma than in patients with severe asthma. 

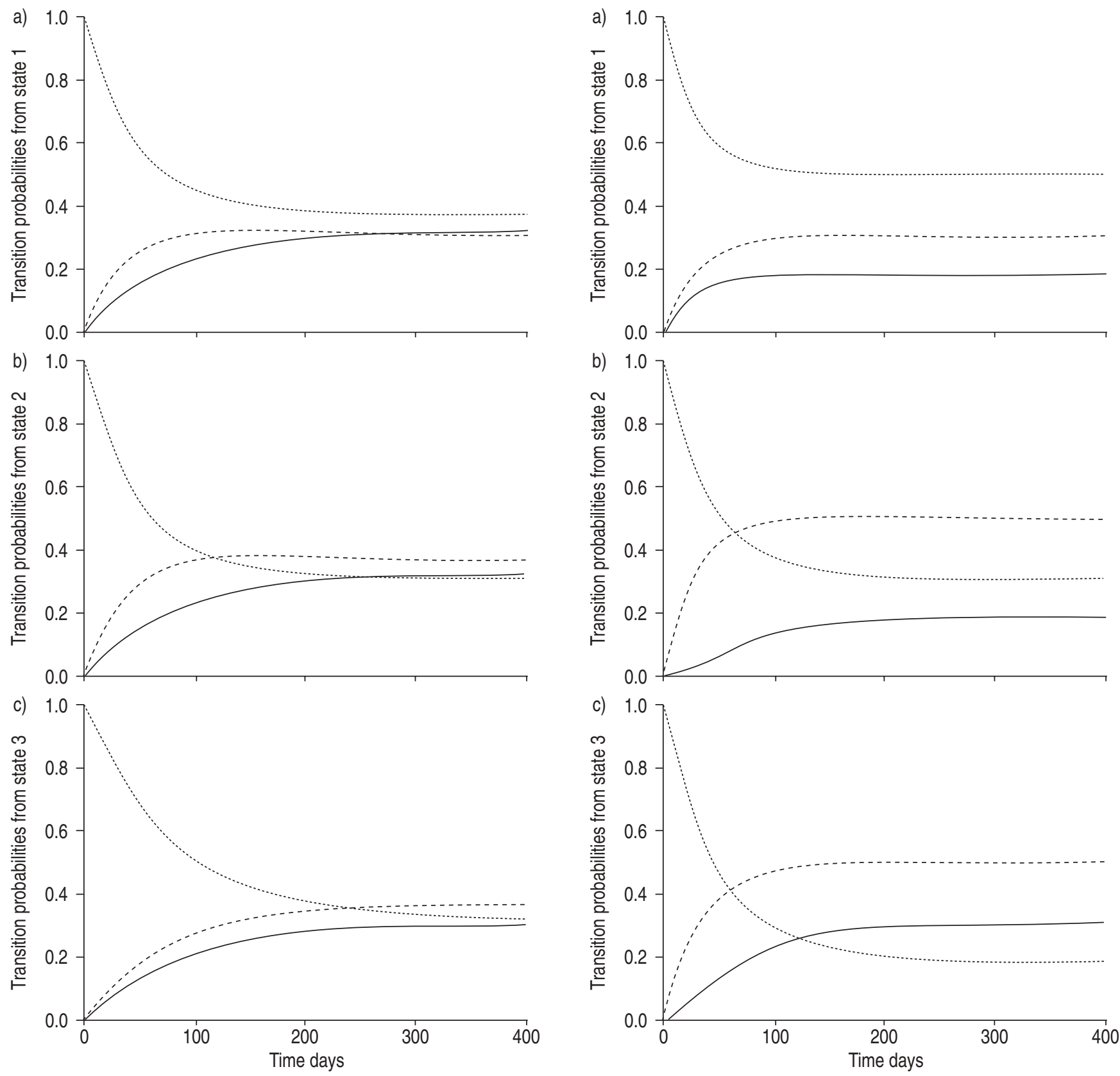

Fig. 2. $-\lambda_{\mathrm{ij}(\mathrm{t})}(\mathrm{i}=1,2,3$ and $\mathrm{j}=1,2,3)$ are the transition probabilities from state "i" to state "j" in time "t". Values of $\lambda_{i j(t)}$ were estimated from the whole study population. Each graph corresponds to a different starting state. In a) ……..: $\lambda_{11(\mathrm{t})} ;--\mathrm{-}_{12(\mathrm{t})} ; \lambda_{3}: \lambda_{13(\mathrm{t})}$, in $\left.\mathrm{b}\right)$ …......: $\lambda_{22(\mathrm{t})} ;---: \lambda_{21(\mathrm{t})} ;-: \lambda_{23(\mathrm{t})}$, and in c) …......: $\lambda_{33(\mathrm{t})} ;--\lambda_{31(\mathrm{t})}$; $-: \lambda_{32(t)}$

Fig. 3. - Values of $\lambda_{\mathrm{ij}(\mathrm{t})}$ were estimated from the patients with mild-tomoderate asthma. Each graph corresponds to a different starting

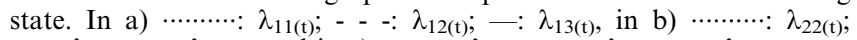
$---: \lambda_{21(t)} ;-: \lambda_{23(t)}$, and in c) ……... $\lambda_{33(\mathrm{t})} ;-\cdots: \lambda_{31(\mathrm{t})} ;-: \lambda_{32(\mathrm{t})}$.

\section{Discussion}

Table 3. - Estimation of transition forces in patients with mildto-moderate or severe asthma and comparison

\begin{tabular}{lrrrrrr}
\hline \multirow{2}{*}{$\begin{array}{l}\text { Transition } \\
\text { forces }\end{array}$} & \multicolumn{2}{c}{ Mild-to-moderate } & & \multicolumn{2}{c}{ Severe } & \multirow{2}{*}{ p-value } \\
\cline { 2 - 3 } \cline { 5 - 6 } & \multicolumn{1}{c}{ Value } & SD & & Value & SD & \\
\hline$\lambda_{12}$ & $10.6 \times 10^{-3}$ & $1.3 \times 10^{-3}$ & & $8.3 \times 10^{-3}$ & $2.5 \times 10^{-3}$ & 0.40 \\
$\lambda_{13}$ & $6.7 \times 10^{-3}$ & $0.8 \times 10^{-3}$ & & $7.7 \times 10^{-3}$ & $1.4 \times 10^{-3}$ & 0.54 \\
$\lambda_{21}$ & $18.6 \times 10^{-3}$ & $2.7 \times 10^{-3}$ & & $4.8 \times 10^{-3}$ & $1.4 \times 10^{-3}$ & $<10^{-3}$ \\
$\lambda_{23}$ & $0.0001 \times 10^{-3}$ & $1.4 \times 10^{-3}$ & & $7.1 \times 10^{-3}$ & $1 \times 10^{-3}$ & $<10^{-3}$ \\
$\lambda_{31}$ & $15.8 \times 10^{-3}$ & $0.5 \times 10^{-3}$ & & $3.2 \times 10^{-3}$ & $0.4 \times 10^{-3}$ & $<10^{-3}$ \\
$\lambda_{32}$ & $2.1 \times 10^{-3}$ & $0.4 \times 10^{-3}$ & $3.8 \times 10^{-3}$ & $0.5 \times 10^{-3}$ & 0.09 \\
\hline
\end{tabular}

This study demonstrates that a Markov model can be developed and used to predict the long-term control of asthma. This model demonstrates the influence of severity on the probability and rate of change between control states. Two findings in particular are noteworthy: 1) patients with mild-to-moderate persistent asthma in suboptimal and unacceptable control had a strong tendency to change directly to optimal control, while patients with severe asthma did not tend to change to any one health state in particular but had difficulty leaving unacceptable control (transition forces are low); 2) the probability of changing control state became stable more quickly (100 days) in patients with mild-tomoderate asthma. 

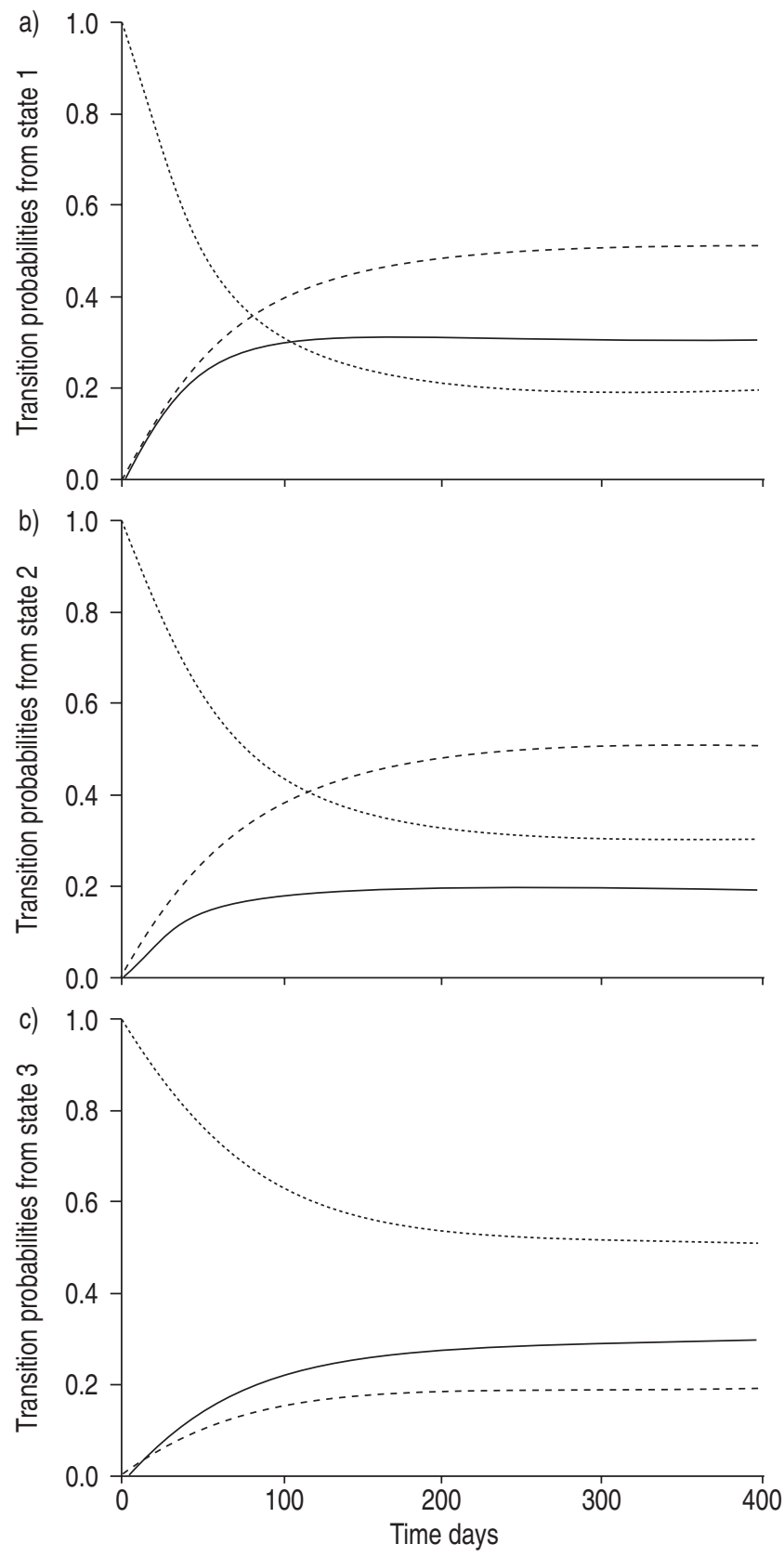

Fig. 4.-Values of $\lambda_{\mathrm{ij}(\mathrm{t})}$ were estimated from the patients with severe asthma. Each graph corresponds to a different starting state. In a) $\cdots \cdots \cdots \cdot \lambda_{11(\mathrm{t})} ;-: \lambda_{12(\mathrm{t})} ;---: \lambda_{13(\mathrm{t})}$, in b) $\cdots \cdots \cdots \cdot \lambda_{22(\mathrm{t})} ;-: \lambda_{21(\mathrm{t})}$; - - - : $\lambda_{23(\mathrm{t})}$, and in c) $\cdots \cdots \cdots \cdot \lambda_{33(\mathrm{t})} ;-\cdots-\lambda_{31(\mathrm{t})} ;-: \lambda_{32(\mathrm{t})}$.

This model allows the time necessary for the state change probability to stabilise, as a function of the degree of severity, and the state of the patient at the time of visit to be predicted. The presented model is really a daily clinical tool if it is used as a concept, that is to say: evaluate the modifications of the control of asthma over time. It also has two clinical interests: firstly, the study shows that a 3-month period is necessary to better establish the phenotype of a patient. Secondly, some factors could modify this period of time. The current paper studied the severity and observed some significant differences between mild-to-moderate and severe asthma. The present authors' believe the concept of the model is interesting and should be known by clinicians. Control of asthma is one of the most commonly used criteria to assess this disease at present. Most patients remained uncontrolled despite therapies and current guidelines advocated for a maximal control. Despite all these statements, there is no single way to define or predict the long-term control of asthma. The current study demonstrates that it can be objectively assessed in a real subset of patients.

One major advantage and originality of the present study is that it deals with data in real time. The patients were not all invited to consult on the same fixed date. The aim was to conduct this study in a naturalistic context of standard practice and to study evolution of asthma control over the course of time.

The tests of the goodness-of-fit of the models were not significant (excepted for the mild-to-moderate asthma at 100 days). Consequently, the homogeneity assumption is not rejected. Severity is an important factor in change of state and stratification was carried out according to the severity. This allowed the transition forces to be compared and the estimators to be improved.

It is currently not possible to estimate the number of patients and transitions necessary to establish a Markov model. In this study, the transition forces associated with change from suboptimal control to unacceptable control was almost null for patients with mild-to-moderate asthma. This is due to the small number of transitions of this type observed in the study. Nevertheless, this transition plays an important role in patients with severe asthma, and has therefore been retained in the model so that patients can be compared according to severity.

Definition or validation of the control states chosen was not the aim of this study. The data in the literature vary on definitions of control states and no established consensus exists. Classification of asthma control in three classes is natural, and a consensus was quickly obtained amongst physicians working with the expert system. There has only been one previous study in the literature that has proposed classifying control at three levels [17], although data from the Asthma Insights and Reality in Europe (AIRE) study [18] also suggest such a classification. In the current study, a classification based on the clinical and functional criteria indicated in the Canadian guidelines [5] was chosen. The data recorded on the clinical report form [13] were sufficiently complete to categorise the patients satisfactorily in this way.

In the absence of consensus, the definition of levels of asthma control is evidently open to discussion. However, the results obtained are neither illogical nor intrinsically unreasonable. For patients with mild-moderate persistent asthma, optimal control appears to be the natural state. The probabilities of transition from suboptimal and unacceptable control to optimal control are high and the mean time to leave optimal control is much longer than for the other two states. Suboptimal control is not a natural end-state, but rather a transition state and therefore unstable. It would be interesting to understand why this is so, and to know whether patients and clinicians are indeed unsatisfied with such control, which is neither perfect nor unacceptable. The state of unacceptable control is interesting to analyse. Patients with mild-to-moderate persistent asthma leave this state quickly, in contrast to patients with severe persistent asthma. For a patient with severe persistent asthma, the transition probability to unacceptable control or of remaining there is very high.

Even though it was not the aim of this study, it seems logical to compare the present results to those of BATEMAN et al. [17]. They showed that an identical proportion of patients were controlled at all levels of severity, and thus do not agree with the observations of the current study which noted a clear effect of asthma severity. This difference between the two studies is probably due to the fact that a therapeutic trial cannot be compared directly with the naturalistic setting of this study. In addition, the levels of severity were probably different in the two studies. 
The originality of this method of analysis is obvious, and the approach has much potential for future studies of the natural history of asthma. The model provides a mathematical tool to evaluate and compare transition forces, and to measure them over the course of time. This model can be applied to real consultation conditions, which vary from one patient to another and from one time to another for the same patient.

Markov models have been used successfully in other chronic conditions, such as lung cancer [19], human immunodeficiency virus (HIV) infection [20, 21], coronary heart disease, hypertension, polyarthritis, and also very recently in bronchial asthma but not with continuous time [22]. At a time when biomarkers (nitric oxide (NO) in exhaled air, level of bronchial hyperreactivity) are being investigated to determine their utility in the management of asthma, the use of this model could allow better prediction and validation of their place according to control and severity. The application of such a Markov model to daily clinical practice could help in decision making and patient information.

Such an approach is important because the concept of control is relevant only if it leads to a therapeutic decision. For example, if control is observed to be optimal for 3 months, management intensity should be reduced if the severity is mild-to-moderate, or maintained if the asthma is more severe. A similar reasoning could be applied to suboptimal and unacceptable control.

Finally, this study could help the clinician motivate certain patients by demonstrating to them the likelihood of improvement with time. The introduction of covariables into the Markov model by adjustment will allow precision of these probabilities tailored to individual patients.

In conclusion, Markov models can be applied to asthma. This model constitutes a tool for clinical evaluations, evaluation of management strategies, and a better adaptation of costs/ efficacy clinical evaluations.

\section{Appendix 1: Definition of the severity}

Severity of asthma has been evaluated for each patient during the first visit. The global evolution of asthma has been taken into account. The different stages of severity were defined as follows. Mild persistent: brief symptom less than once a day, no severe exacerbation, forced expiratory volume in one second (FEV1) $>80 \%$ pred, inhaled beclomethasone dipropionate $(\mathrm{BDP}) \leqslant 500 \mu \mathrm{g}$; Moderate persistent: brief symptoms daily, nocturnal symptoms once a week, 1-2 severe exacerbations, FEV1 between $60-80 \%$ pred, inhaled BDP between 500-1,500 $\mu \mathrm{g}+$ long acting $\beta_{2}$-agonist; Severe persistent: brief symptoms daily, dyspnoea limits activities, nocturnal symptoms frequent, exacerbation $\leqslant 2$, one exacerbation requiring emergency room or hospitalisation, FEV $1 \leqslant 60 \%$ pred, inhaled BDP $\geqslant 1,500 \mu \mathrm{g}+$ long-acting $\beta_{2}$-agonist+oral dose of glucocorticoids.

\section{Appendix 2: Definition of the states}

A control state was allocated to each patient at each consultation. The overall control state chosen was defined as the highest of the states determined for each of the following individual variables: exacerbation, $\beta_{2}$-mimetic use associated with the presence of symptoms, measurement of FEV1 and dyspnoea.

Definition of exacerbation: the variables which related to exacerbations were "E-Type" (nature of the episodes) and "E-corticoid" (corticoid use). The various levels of "E-Type" variable were: none; instability; attack; serious attack at home; serious attack requiring hospitalisation; serious attack requiring intensive care. The various levels of the variable "E-corticoid" were: false and true. When "E-Type" was coded "serious attack..." and "E-corticoid" was coded "true", then the patient was classed in state 3 .

When "E-Type" was coded "serious attack..." and "E-corticoid" was coded "false", then the patient was classed in state 2 . In all other cases, the patient was classed in state 1 .

$\beta_{2}$-mimetic use associated with the presence of symptoms: The variables "entry- $\beta_{2}$-spray-dose" and "entry- $\beta_{2}$-spray-unit" gave information about the quantity of $\beta_{2}$-mimetics used. The presence of a symptom was obtained by the variable "attackfrequency-recent". The variable "entry- $\beta_{2}$-spray-dose" was quantitative (range: $0-30$ ).

The various grades of the variable "entry- $\beta_{2}$-spray-unit" were: puffs per week and puffs per day. The various grades of the variable "attack-frequency-recent" were: absent; one per month; <1 per week; 1 per week; several per week; 1 per day; several per day. Patients were classed into health states in the following way: 1) if the quantity of $\beta_{2}$ used was greater (or equal) to one puff per day and if the frequency of attacks was greater (or equal) to 1 per day, the patient was classed in state $3 ; 2$ ) if the patient did not fulfil the conditions to be classed in state 3 and if the quantity of $\beta_{2}$-agonist used was greater (or equal) to 1 puff per week and if the frequency of attacks was greater (or equal) to 1 per week, then the patient was classed in state $2 ; 3)$ in all other cases the quantity of $\beta_{2}$-agonist used was $<1$ puff per week or if the frequency of attacks was $\leqslant 1$ per week, the patient was classed in state 1 .

Measurement of FEV1: the variable corresponding to this criteria was, "FEV1-baseline-relationship". This indicates the relationship between measured FEV1 and predicted FEV1. A decrease of $>30 \%$ in the measured FEV1 compared with the maximum FEV1 measured for a patient led to the patient being classed in state 3 , a decrease of $10-30 \%$ to state 2 , and if no decrease was observed the patient was classed in state 1 .

Dyspnoea: the variables corresponding to this criterion were "recent-dyspnoea-exertion" and "recent-dyspnoea-between attacks". The grades of the variable "recent-dyspnoea-exertion" were: absent, violent exertion, rapid walk, normal walk, less exertion. "Recent-dyspnoea-between attacks" was scored as: true or false. When "recent-dyspnoea-between attacks" was coded "true", the patient was classed in state 3 . When "recent-dyspnoeabetween attacks" was coded "false": 1) if "recent-dyspnoea-exertion" was coded "minimum effort" or "normal pace", the patient was classed in state 3 ; 2) if "recent-dyspnoea-exertion" was coded "rapid pace", the patient was classed in state 2;3) if "recentdyspnoea-exertion" was coded "violent effort" or "absent", the patient was classed as state 1 .

\footnotetext{
Members of the Association pour la Recherche en Intelligence Artificielle ${ }^{T M}$ (ARIA) group (France). J.P. Orlando (Aubagne), A. Prud'homme (Tarbes), B. Wallaërt (Lille), A. Taytard, A. Magnan, D. Vervloët (Marseille), D. Dusser, N. Dufeu (Paris).
}

\section{References}

1. National Institutes of Health. Expert Panel Report 2: Guidelines for the diagnosis and management of asthma. NIH publication no. 97-4051. Bethesda, National Institutes of Health/National Heart, Lung and Blood Institute, 1997.

2. Cluzel M, Damon M, Chanez P, et al. Enhanced alveolar cell luminol-dependent chemiluminescence in asthma. J Allergy Clin Immunol. 1987; 80: 195-201.

3. Bousquet J, Chanez P, Lacoste JY, et al. Eosinophilic inflammation in asthma. N Engl J Med 1990; 323: 10331039. 
4. Bentley AM, Menz G, Storz C, et al. Identification of $\mathrm{T}$ lymphocytes, macrophages, and activated eosinophils in the bronchial mucosa in intrinsic asthma. Relationship to symptoms and bronchial responsiveness. Am Rev Respir Dis 1992; 146: 500-506.

5. Boulet LP, Becker A, Berube D, et al. Canadian Asthma Consensus Report, 1999. Canadian Asthma Consensus Group. CMAJ 1999; 161: Suppl. 11, S1-61.

6. Godard P, Clark TJ, Busse WW, et al. Clinical assessment of patients. Eur Respir J 1998; 11: Suppl. 26, 2S-5S.

7. Cockcroft DW, Swystum VA. Asthma control versus asthma severity. J Allergy Clin Immunol 1996; 98: 1016-1018.

8. Reddel H, Jenkins C, Woolcock A. Diurnal variability-time to change asthma guidelines? BMJ 1999; 319: 45-47.

9. Sont JK, Willems LN, Bel EH, et al. Clinical control and histopathologic outcome of asthma when using airway hyperresponsiveness as an additional guide to long-term treatment. The AMPUL Study Group. Am J Respir Crit Care Med 1999; 159: 1043-1051.

10. Jatakanon A, Lim S, Barnes PJ. Changes in sputum eosinophils predict loss of asthma control. Am J Respir Crit Care Med 2000; 161: 64-72.

11. Juniper EF, O'Byrne PM, Guyatt GH, Ferrie PJ, King DR. Development and validation of a questionnaire to measure asthma control. Eur Respir J 1999; 14: 902-907.

12. Vollmer WM, Markson LE, $\mathrm{O}^{\prime}$ Connor E, et al. Association of asthma control with health care utilization and quality of life. Am J Respir Crit Care Med 1999; 160: 1647-1652.

13. Redier H, Daures JP, Michel C, et al. Assessment of the severity of asthma by an expert system. Description and evaluation. Am J Respir Crit Care Med 1995; 151: 345-352.
14. Kalbfleish JD, Lawless JF. The analysis of panel data under a Markov assumption. J Am Stat Assoc 1985; 80: 863871.

15. Boudemaghe T, Daurès JP. Modélisation de l'asthme par un modèle multi-état. Rev Epidemiol Santé Publique 2000; 48: 249-255.

16. Kotz S, Johnson NL, Read CB. Encyclopedia of Statistical Sciences. Vol 8. New York, Wiley-Intersciences, 1988; pp. 646-647.

17. Bateman ED, Bousquet J, Braunstein GL. Is overall asthma control being achieved? A hypothesis-generating study. Eur Respir J 2001; 17: 589-595.

18. Rabe KF, Vermeire PA, Soriano JB, Maier WC. Clinical management of asthma in 1999: the Asthma Insights and Reality in Europe (AIRE) study. Eur Respir J 2000; 16: 802807.

19. Boher JM, Pujol JL, Grenier J, Daurès JP. Markov model and markers of small cell lung cancer: assessing the influence of reversible serum NSE, CYFRA 21-1 and TPS levels on prognosis. Brit J Cancer 1999; 79: 1419-1427.

20. Gentleman RC, Lawless JF, Lindsey JC, Yan P. Multi-state Markov models for analysing incomplete disease history data with illustrations for HIV disease. Stat Med 1994; 13 : 805-821.

21. Longini IM, Clark WS, Byers RH, et al. Statistical analysis of the stages of HIV infection using a Markov model. Stat Med 1989; 8: 831-843.

22. Paltiel AD, Fuhlbrigge AL, Kitch BT, et al. Cost-effectiveness of inhaled corticosteroids in adults with mild-to-moderate asthma: results from the Asthma Policy Model. J Allergy Clin Immunol 2001; 108: 39-49. 